\title{
Research Paper: Comparison of Shoulder Proprioception in Women with and without Generalized Joint Laxity
}

\author{
Mahan Rastgar ${ }^{1},{ }^{*}$ Afsun Nodehi Moghadam ${ }^{1}$, Enayatollah Bakhshi ${ }^{1}$, Elham Sarabadani Tafreshi ${ }^{1}$, Sahar Toluee ${ }^{1}$
}

1. Department of Physiotherapy, University of Social Welfare and Rehabilitation Sciences, Tehran, Iran.

Cftat On: Rastgar M, Nodehi Moghadam A, Bakhshi E, Sarabadani Tafreshi E, Toluee S. [Comparison of Shoulder Proprioception in Women with and without Generalized Joint Laxity (Persian)]. Journal of Rehabilitation. 2016; 17(2):128-135. http://dx.crossref.org/ 10.21859/jrehab-1702128

: http://dx.crossref.org/ 10.21859/jrehab-1702128

Received: 14 Mar. 2016 Accepted: 01 Jun. 2016

\section{ABSTRACT}

Objective Generalized joint hypermobility predisposes some individuals to a wide variety of musculoskeletal complaints, especially in the shoulder joint. Proprioception, which includes joint position sense and sense of movement, has an important role in the functional stability of shoulder joint. Given the critical role of proprioception in the functional stability of shoulder, the primary aim of this study was to compare shoulder joint position sense and sense of movement (kinesthesia) between individuals with and without general joint hyper mobility. The secondary aim of this study was to compare proprioception between dominant and non-dominant sides in each group.

Materials \& Methods In this causal-comparative study, 20 females with generalized joint laxity (Mean[SD] age $=22.05[2.30] \mathrm{y}$ ) and 20 females without generalized joint laxity (Mean[SD] age=22.65[2.53] y) participated in the study. Testing was performed in the supine position. Prior to beginning each proprioception tests, the participants were given to practice trials to become familiar with the testing procedure. Proprioception tests were performed during passive repositioning and kinesthesia using an isokinetic dynamometer. The shoulder external rotation range was measured with a standard goniometer, and $90 \%$ of its range was considered as a target angle in passive reposition test. The kinesthetic sense of the shoulder was evaluated by measuring the threshold for passive external rotation. The speed of threshold to detection of passive motion test was at $0.5 \mathrm{deg} / \mathrm{s}$ and passive reproduction of joint position was at $2 \mathrm{deg} / \mathrm{s}$. Both dominant and non-dominant sides of all the subjects were evaluated. Test sequences for measuring the threshold to detection of passive motion and passive reproduction of joint position, as well as dominant and non-dominant shoulder were random. The blindfold and headphones were used to remove visual and auditory feedback. The mean of three repetitions of passive reproduction of target angle and threshold to detection of passive motion were calculated. Independent t-test was used to compare joint position sense and kinesthesia between females with and without generalized joint laxity, and paired $t$ test was used to statistically analyze differences between dominant and non-dominant sides in each group.

Results No significant difference was observed in joint position test acuity and of detection of motion in each of dominant and non-dominant sides between females with and without generalized joint laxity (dominant side: $\mathrm{P}=0.47$, non-dominant: $\mathrm{P}=0.70$ ). Females with generalized joint laxity exhibited significantly larger errors in passive joint reproduction tests in both dominant and non-dominant sides $(P=0.001)$ compared with those without generalized joint laxity.

Conclusion The results revealed that the angle repositioning sense in the extreme range of shoulder joints in females with generalized joint laxity is reduced compared to those without generalized joint laxity. Lower joint position test acuity in females with generalized joint laxity may relate to the disturbance or loss of sensory messages from joint receptors to the central nervous system. Reduced proprioception feedback may lead to biomechanically unsound limb positions being adopted. Such a mechanism may allow acceleration of degenerative joint conditions and may account for the increased prevalence of musculoskeletal complaints seen in subjects with generalized joint laxity.
Keywords: Generalized joint laxity, Shoulder joint, Proprioception

\section{* Corresponding Author:}

Afsun Nodehi Moghadam, PhD

Address: Department of Physiotherapy, University of Social Welfare and Rehabilitation Sciences, Koodakyar Ave., Daneshjoo Blvd., Evin, Tehran, Iran. Tel: +98 (21) 22180039

E-Mail: afsoonnodehi@gmail.com 


\title{
مقايسه حس عمقى مفصل شانه در زنان با و بدونشلى عمومى مفاصل
}

\author{
ماهان رستكار'، "|فسون نودهىمقدم'، عنايتالله بخشى'، الهام سر آبادانىتفرشى'، سحر طلوعى'
}

1- كروه فيزيوترايى، انشكاه علوم بيزيستى وتوانبخشى، تهران، ايران.

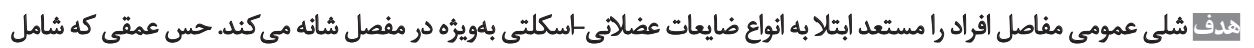

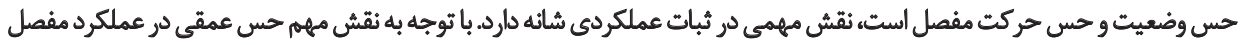

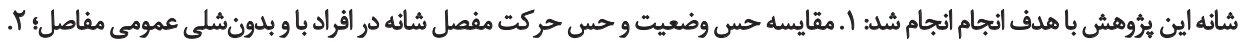

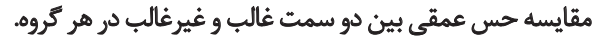

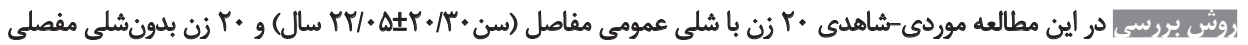

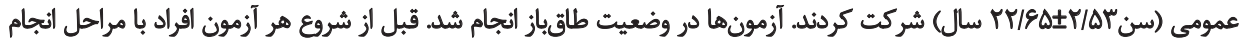

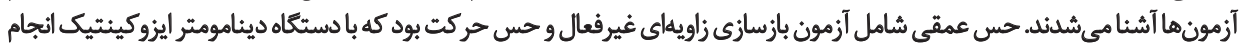

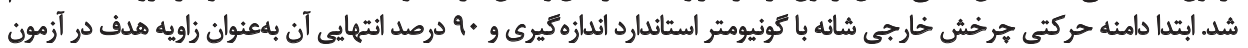

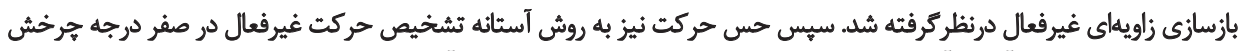

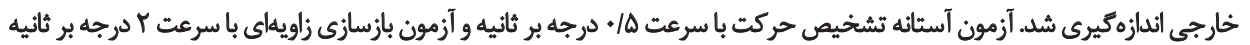

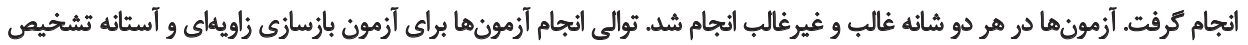

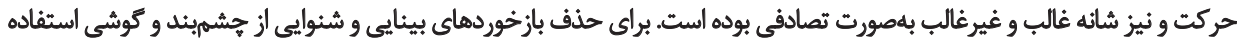

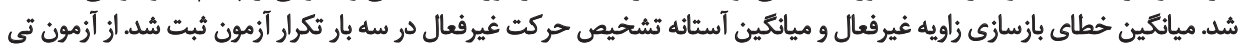

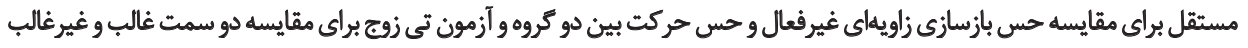

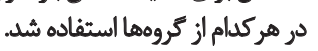

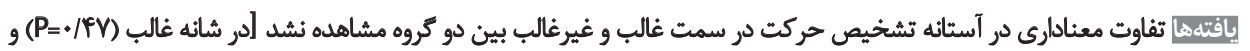

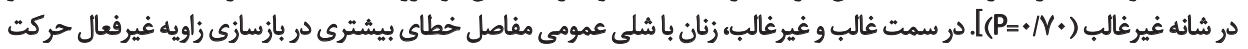

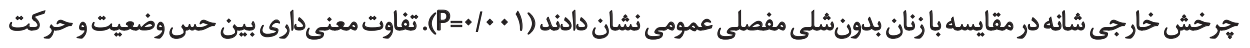

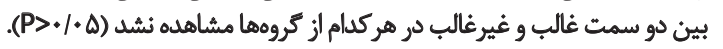

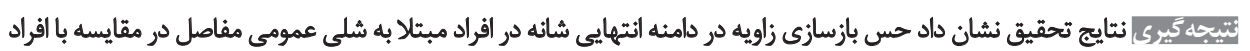

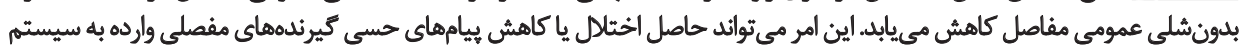

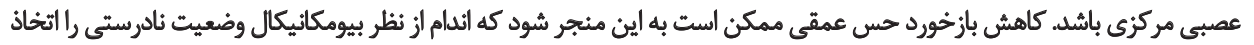

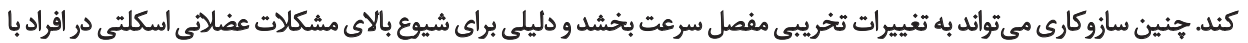

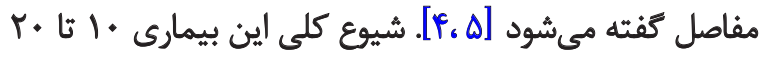

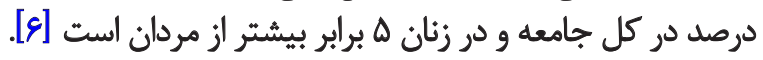

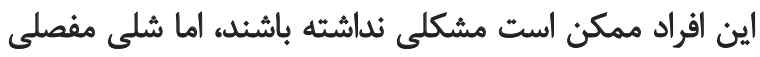

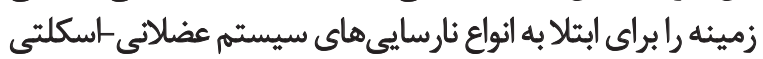
فراهم مي كند [ن]

شانه يكى از مفاصلى است كه در اين افراد دجار ضايعات

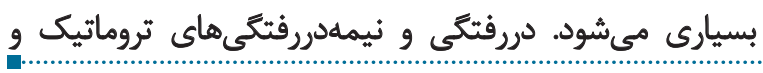

شلى عمومي مفاصل بهمعناي افزايش دامنه حركتى بيشازئحد

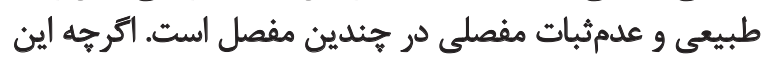

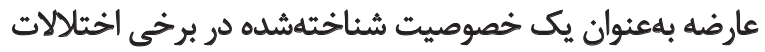

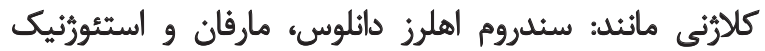

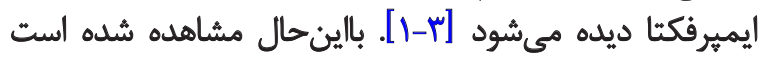

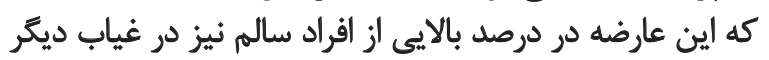

$$
\begin{aligned}
& \text { : نويسنده مسئول: } \\
& \text { دكتر افسون نودهئمقدم: }
\end{aligned}
$$


شانه در اين افراد كمك مي كند.

روش بروسي

در اين مطالعه مورد-شاهدى، •r خانم از دانشجويان دانشكاه

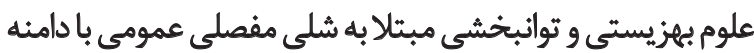

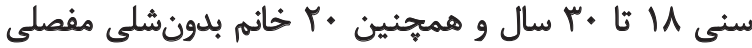

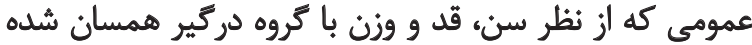

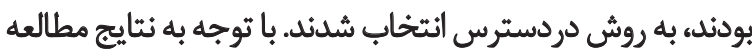

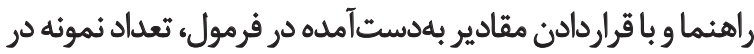

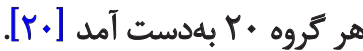

$$
n=\frac{\left[z_{1-0 / 2}+z_{\beta}\right]\left[\sigma_{1}^{1}+\sigma_{2}^{2}\right]}{d^{2}}=\frac{(1.94+0.84)^{2}\left((0.48)^{2}+(0.54)^{2}\right)}{(0.5)^{2}} 16.37=17
$$

معيارهاي ورود افراد به مطالعه عبارت بود از: شاخص بيتون

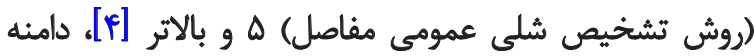

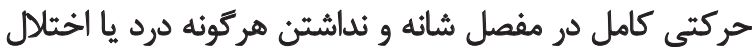

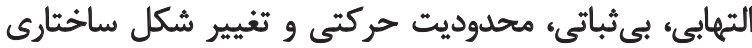

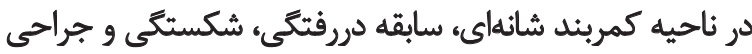

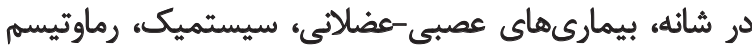

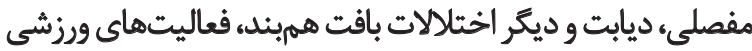

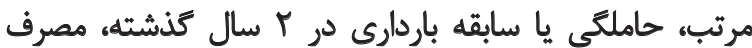

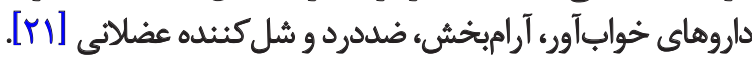

بعد از كرفتن رضايتنامه آكاهانه و كتبى و تكميل يرسش خامنامه

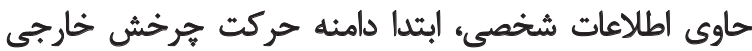

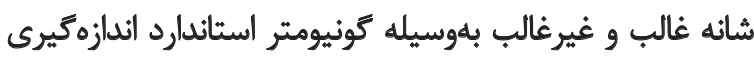

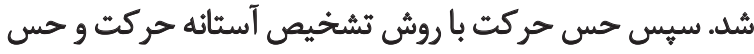
وضعيت با روش بازسازى زاويداى غيرفعال با استفاده از دستئكاه

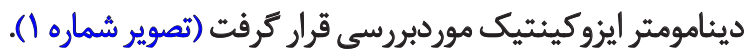

اندازوة كيرى دامثله انتهايى حركتى هرخش خارجى شانه

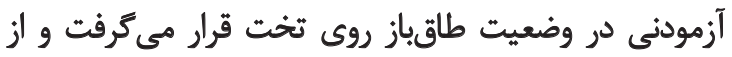

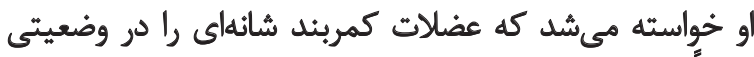

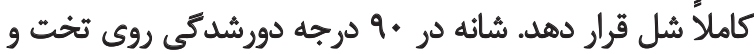

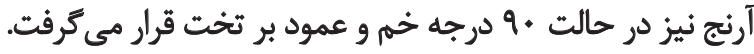

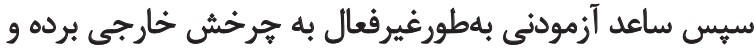

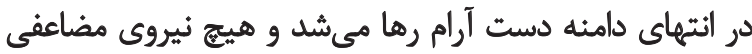

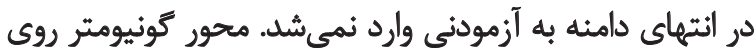

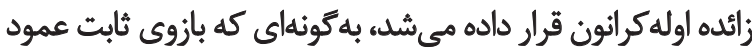

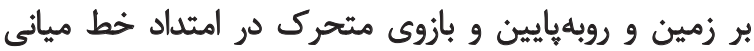

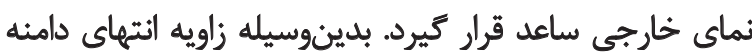

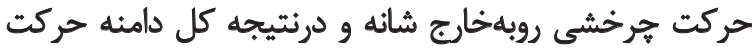

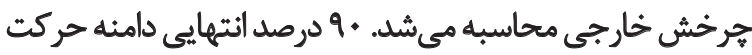
هرخش خارجى بهعنوان زاويه هدف در آزمون بازسازى زاويداى دائ

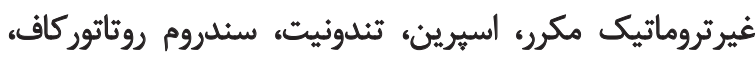

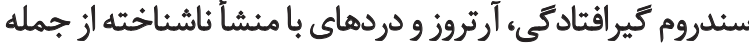

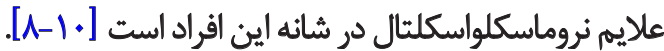

ثبات عملكردى شانه حاصل ارتباط متقابل بين ثبات إنبات

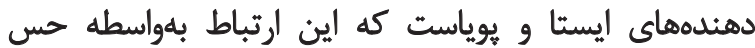

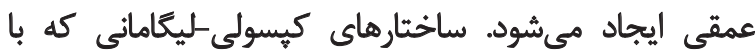

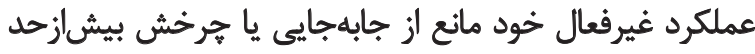

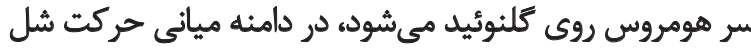

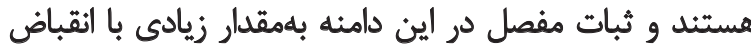

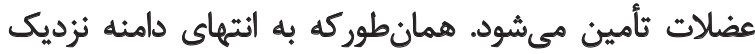

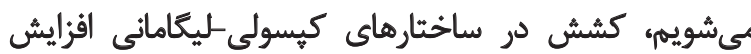

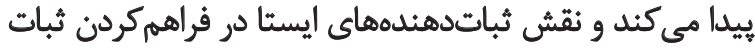

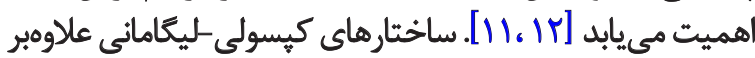

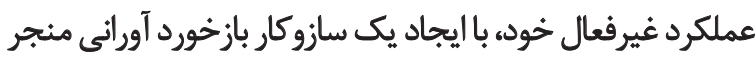

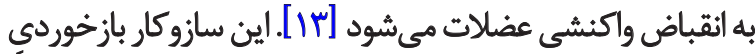

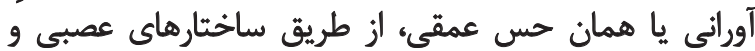

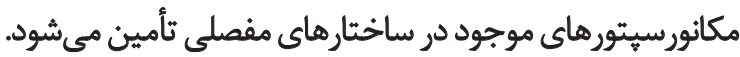
افزايش فشار در ساختارهاى كيسولى لـيكامانى در انتهاي

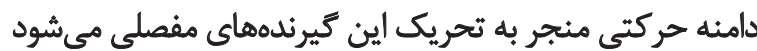

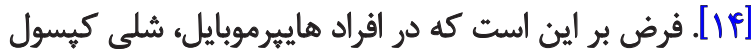

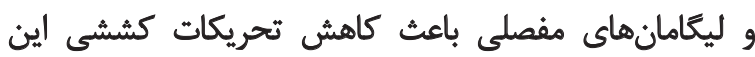

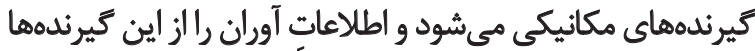

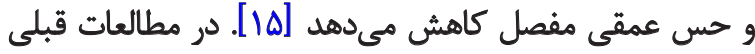

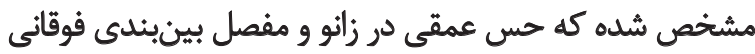

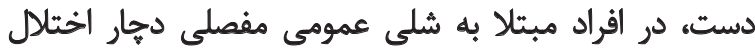

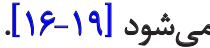

تاكنون هيج مطالعهاى به بررسى حس عمقى مفصل شانه در

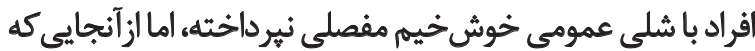

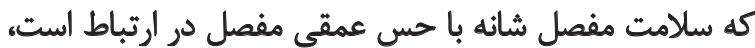

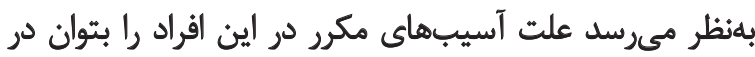

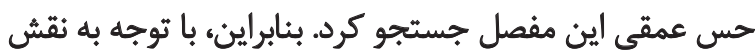

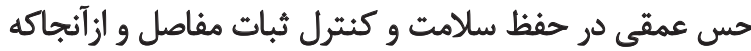

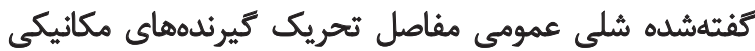

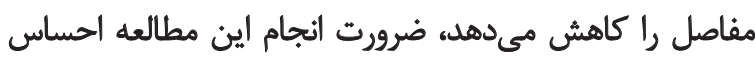

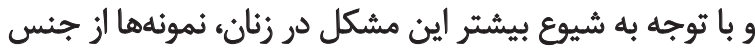

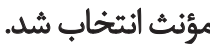

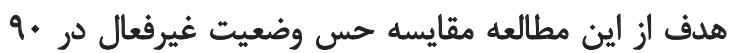

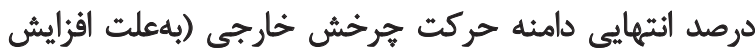

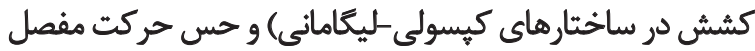

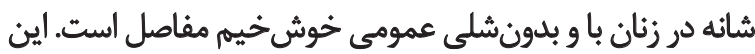

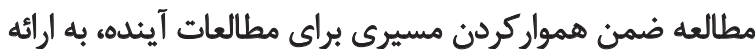

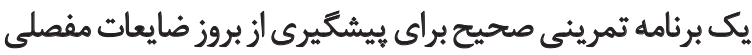




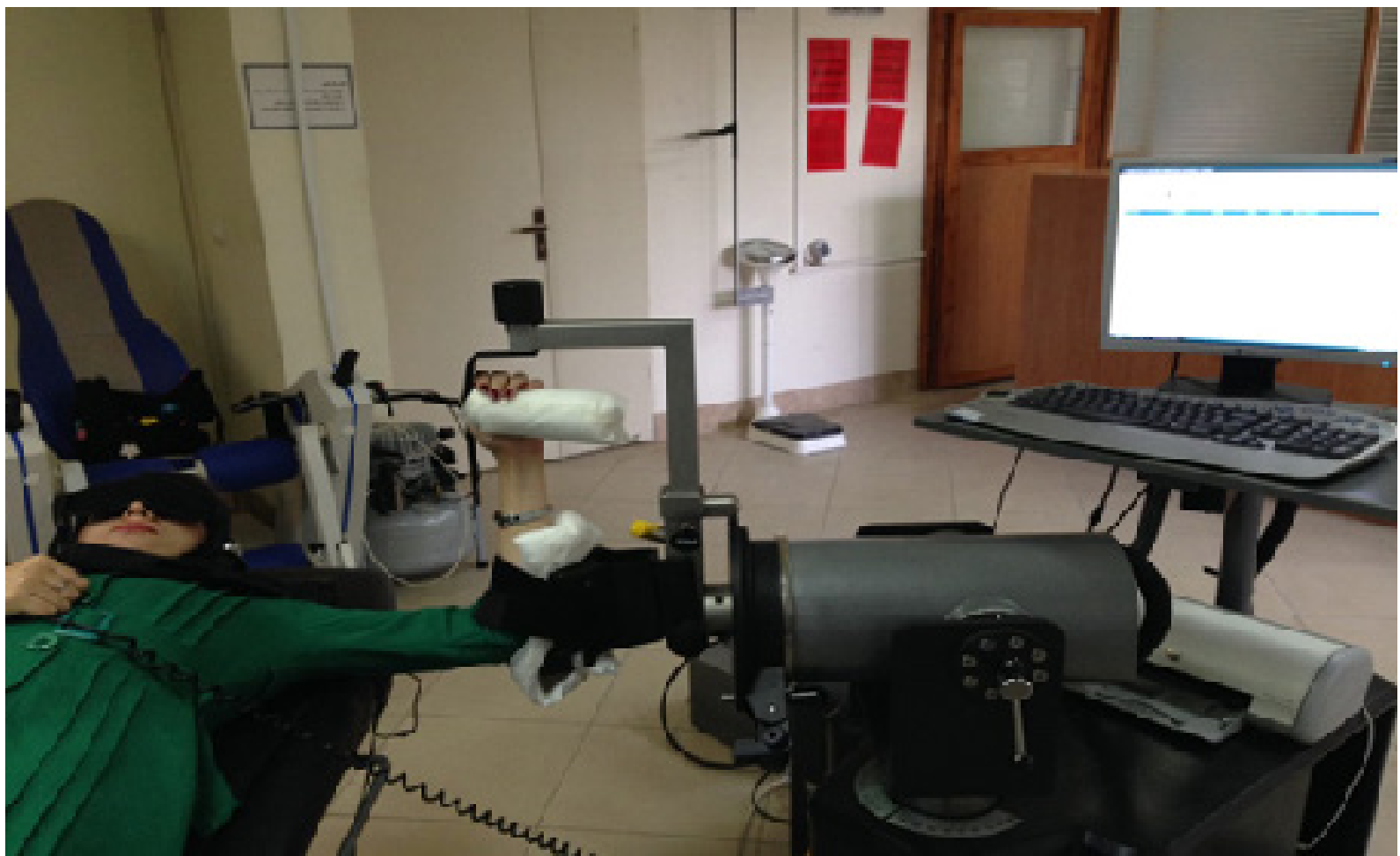

توانبخننى

تصوير ا. روش انجام آزمون حس عمقى با استفاده از سيستم دينامومتر ايزوكينتيك.

$$
\text { آز آزون آستائه تشخيص حركت }
$$

درنظر

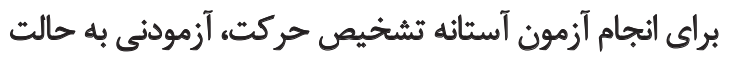

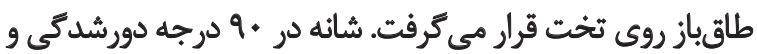

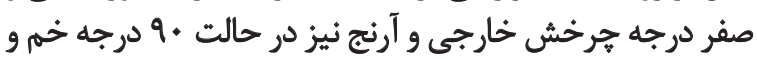

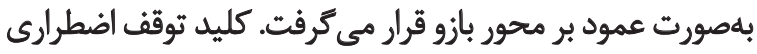

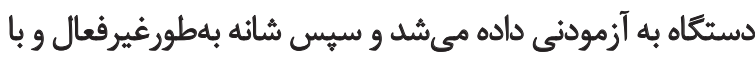

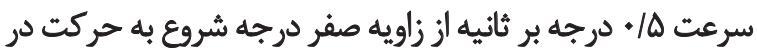

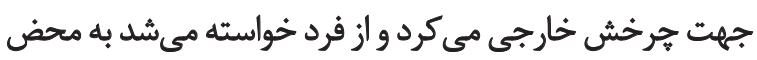

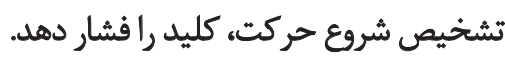

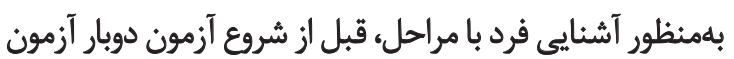

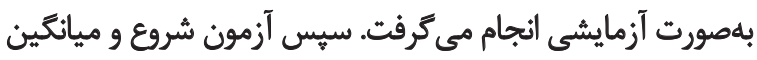

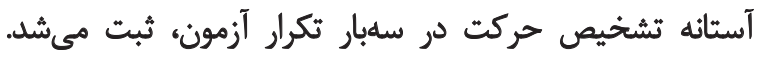

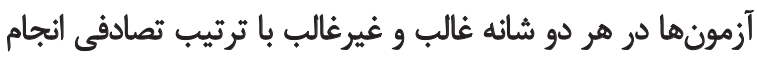

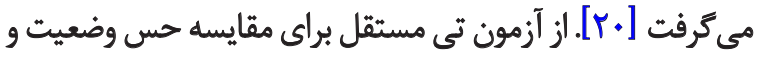

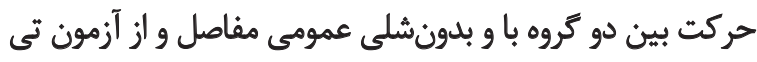

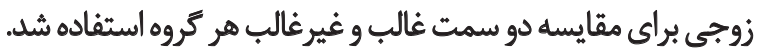

يافتهها

مشخصات جمعيتشناختى و متغيرهاى زمينهاى نمونهاي

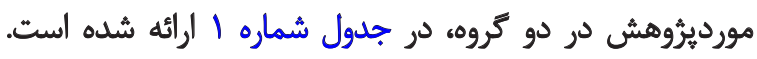

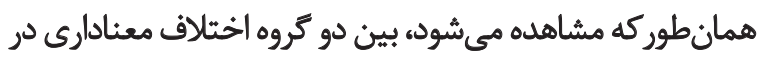

$$
\text { آزمون بازسازى زاويه }
$$

براى انجام آزمون بازسازي زاويهاى غيرفعال، آزمودنى در

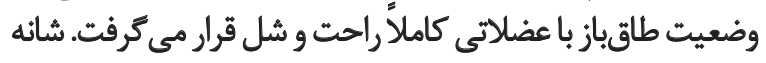

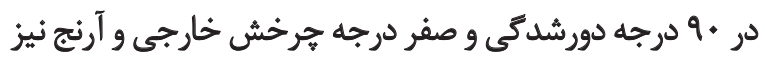

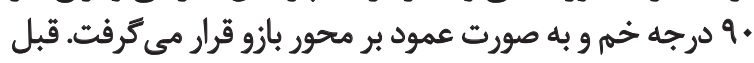

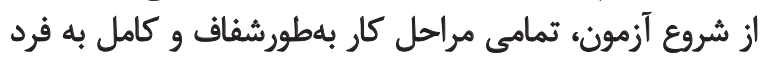

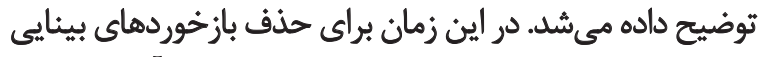

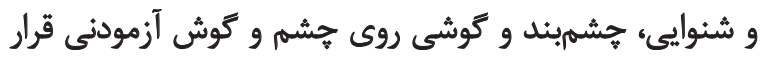

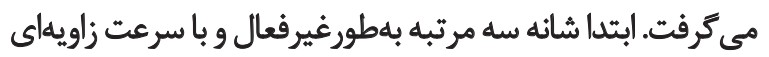

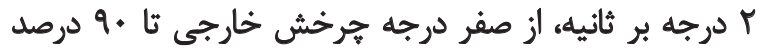

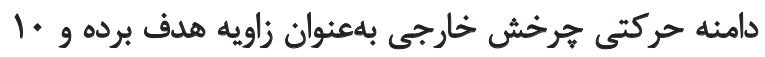

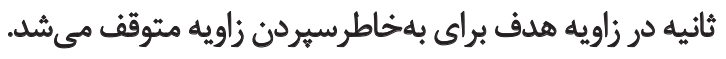
بعد از اين مرحله، كليد توقف اضطرارى دستكاه به آزمودنى

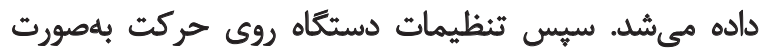

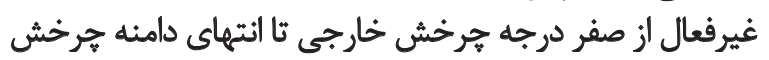

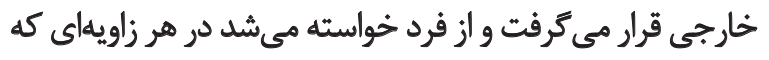

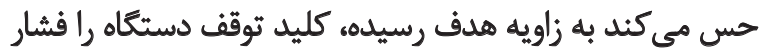

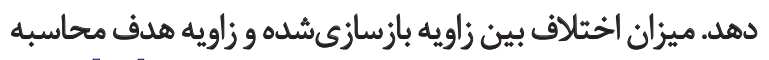

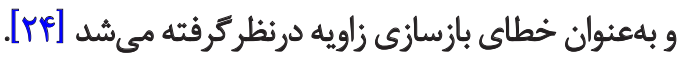


جدول ا. مقايسه مشخصات جمعيتشناختى در دو كروه با و بدونشلى عمومى مفاصل براساس آزمون تى مسيتقل.

\begin{tabular}{|c|c|c|c|}
\hline \multirow{2}{*}{ P-value } & \multicolumn{2}{|c|}{ ميانكين (انحراف معيار) } & \multirow{2}{*}{ متغير (واحد اندازهتيرى) } \\
\hline & با شيلي مفحلي عمومي & بدون شلى عمومى مفصلى & \\
\hline.$/ 48$ & $M / \circ \Delta\left(Y / T_{+}\right)$ & MT/EQ(Y/AT) & سن (سال) \\
\hline 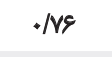 & $\mid E T / \Delta \Delta(F / M T)$ & $\mid \& T / \Lambda \cdot(\Delta / T \mid)$ & قد (سائتى متر) \\
\hline.$/ A V$ & $\Delta V /+\Delta(q / e q)$ & $\Delta \& / F \cdot(8 / 9 Y)$ & وزن (كيلوكرم) \\
\hline.$/ \Delta V$ & TV/AV(T/MQ) & PVITI(NA) & شاخص توده بدنى (كيلوكرم/مترمربع) \\
\hline
\end{tabular}

توانبخننى

جدول r. نتايج آزمون تى مستقل براى مثايسه ميانتين آستانه تشخيص حركت و خطاى بازسازى زاويعاى در دو كروه باو بدونشلى مفصلى عمومى.

\begin{tabular}{|c|c|c|c|c|}
\hline P-value & كروه بدونشلى عمومى مفصلى & كروه با شلى مفصلى عمومى & سمت & متغير \\
\hline$+/ 4 V$ & $1 / \wedge 1 \pm+/ 19$ & $r / *+ \pm * / 9 q$ & غالب & \multirow{2}{*}{ آستائه تشخيص حركت (درجه) } \\
\hline$+N+$ & V/FAー+AT & $1 / 79 \pm+/ 19$ & غيرغالب & \\
\hline $.1 . .1$ & $r / \lambda \digamma \pm 1 / \lambda$. & $N N= \pm \Delta / \cdot r$ & غالب & \multirow{2}{*}{ خطايى بازٔسازى زاويهاى (درجه) } \\
\hline $.1 .+1$ & $\varphi / N^{\prime} \pm 1 / n$ & NEY $\pm F / F V$ & غعيرغالب & \\
\hline
\end{tabular}

توانبخننى

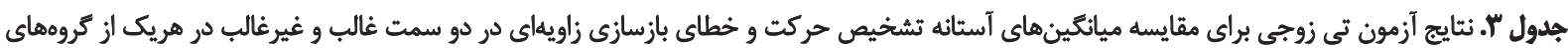
با و بدون شلى مفصلى عمومى.

\begin{tabular}{|c|c|c|c|c|}
\hline \multirow{2}{*}{ P-value } & \multicolumn{2}{|c|}{ سمت } & \multirow{2}{*}{ متغير } & \multirow{2}{*}{ تروه } \\
\hline & غيرغالب & غالب & & \\
\hline - /Ar & $V / A F \pm=/ A T$ & $\mid / / 1 \pm \cdot / A 9$ & آستانه تشخيص حركت (درجه) & \multirow{2}{*}{ بدونشلى عمومى مفاصل } \\
\hline . /ar & $r / M \pm M$ & $P / P A \pm V / A$. & خطاى بازسازى زاويهاى (درجه) & \\
\hline$+/ 94$ & $1 / 9 r \pm \cdot / v q$ & $r / r \pm+/ q q$ & أستائه تشخيص حركت (درجه) & \multirow{2}{*}{ با شلى عمومى معاصل } \\
\hline.$/ W$ & NEYIFIPV & $N N \pm \mathbb{A} /+r$ & هطاي بازٔسازيى زاويهايى (درجه) & \\
\hline
\end{tabular}

توانبخننى

موارد مذكور وجود نداشت و كروهها همكن بودند. بين ميانكين بحث نتايج تحقيق حاضر نشان داد كه تفاوتى بين آستانه تشخيص

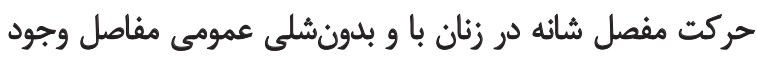

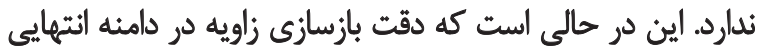

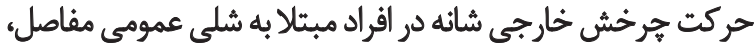
كمتر از افراد بدونش شلى عمومى مفاصل درافل است.

در اين يُوهش، آزمون بازسازى زاويه در دامنه انته انتهايى

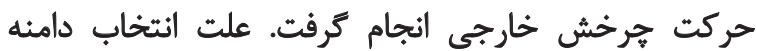

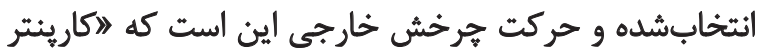

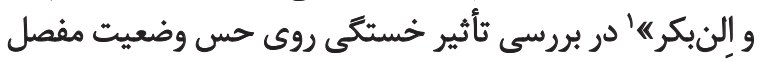

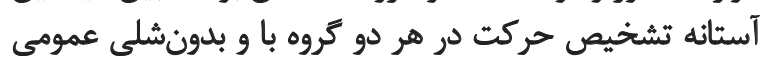

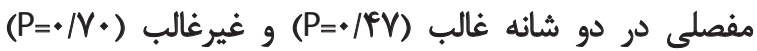

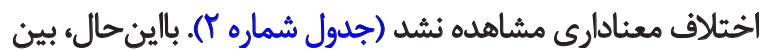

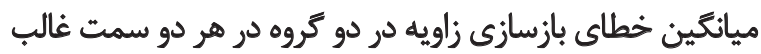

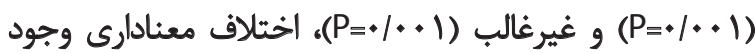

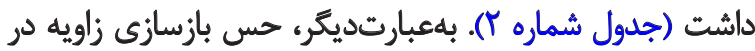

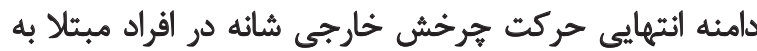

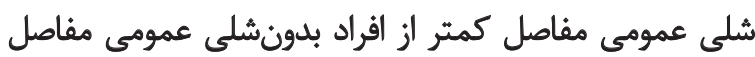

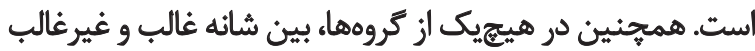

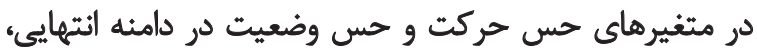

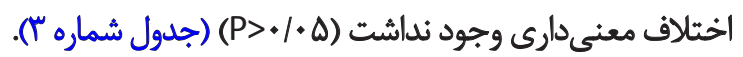


بهنظر مى رسد كه در آزمون آسئانه تشخيص حركت كه حس شروع

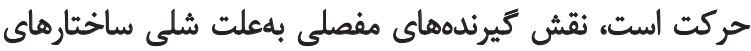

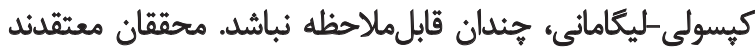

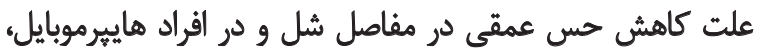

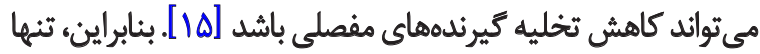

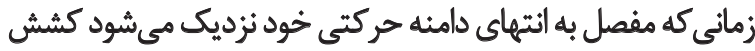

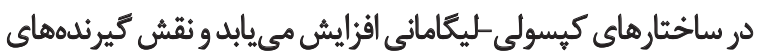

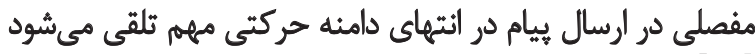

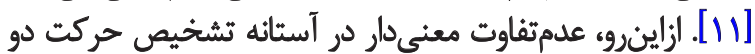

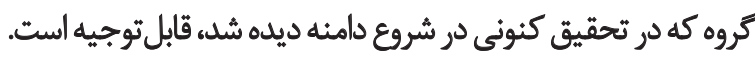

يافته ديكر تحقيق حاضر اين بود كه دقت حس وضعيت

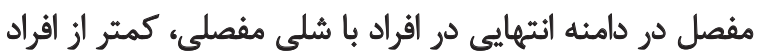

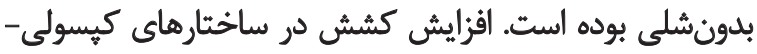

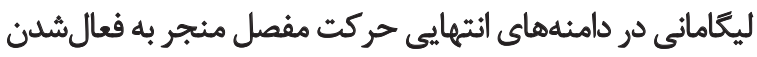

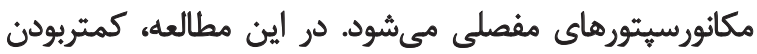

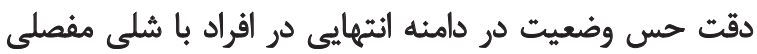

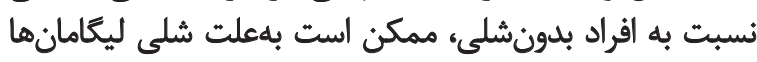

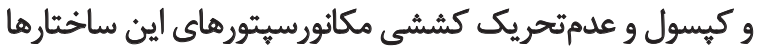

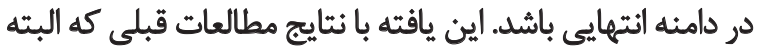

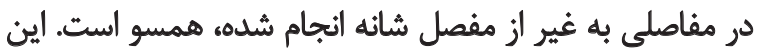

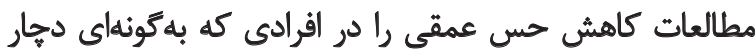

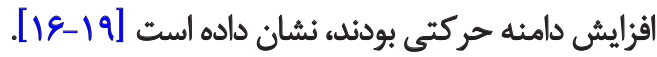

درحقيقت اين تحقيقات مدعى است علت كاهش حس عمقى إسى

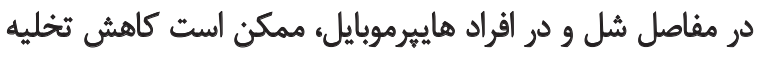

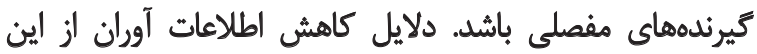

كيرندهها مي ثواند در قالب سه فرضيد ملايل مطامث شود:

• براساس فرضيه اول، نقص در حس عمقى مفصل -كه

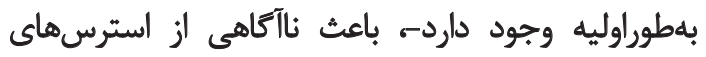

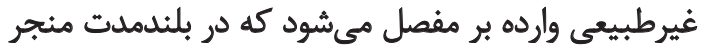

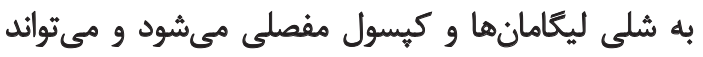

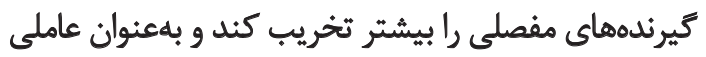
باعث اختلال بيشتر حس عمقى شود [1 19]؛

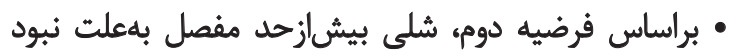

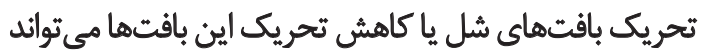

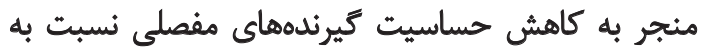

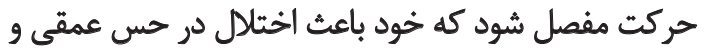

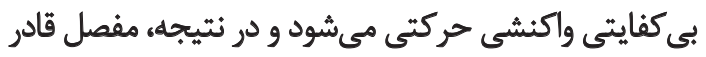

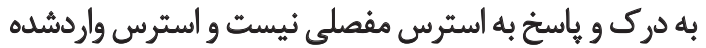

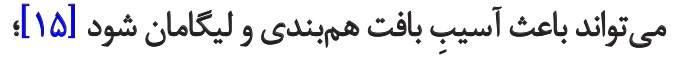
• براساس فرضيه سوم، افزايش تحرك در مفاصل زمينه تخريب

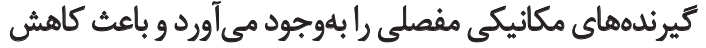

شانه به اين نتيجه رسيدند كه كيرندههاى عضلات جران خانئنده

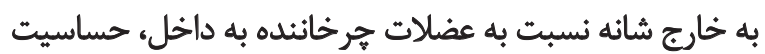

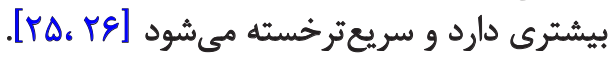

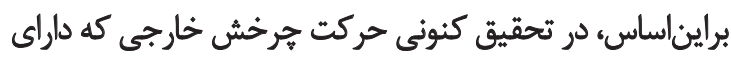

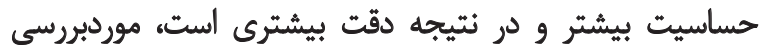

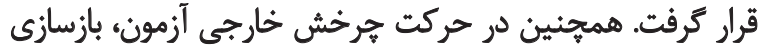

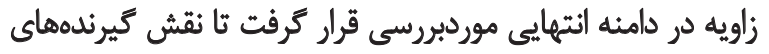

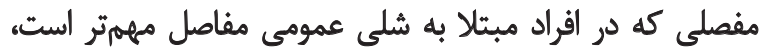

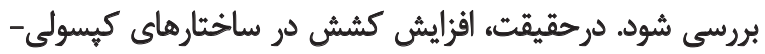

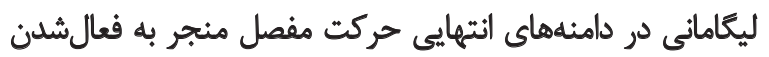

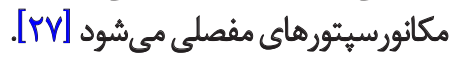

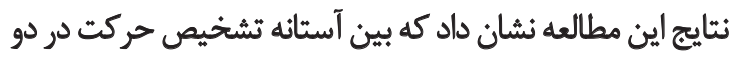

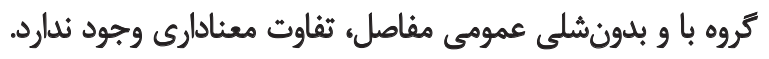

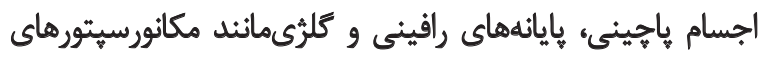

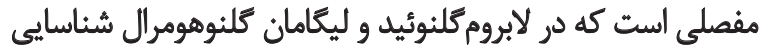

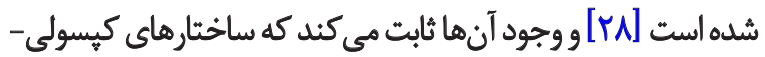

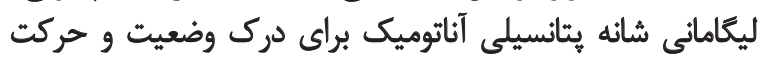

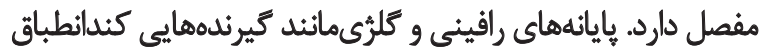

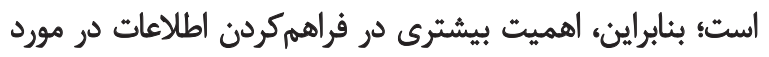

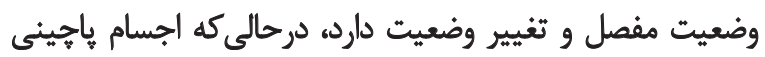

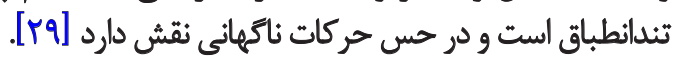
مطالعات نشان دادهاست كيرندهاى كَيسولار تنهادر انتهاى دامنه

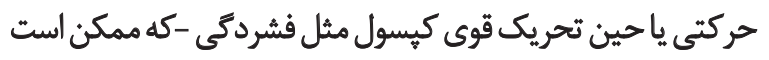

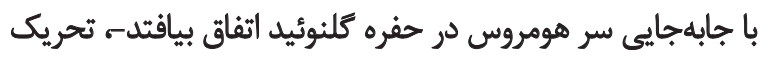

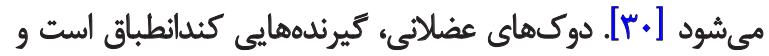

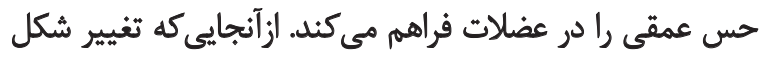

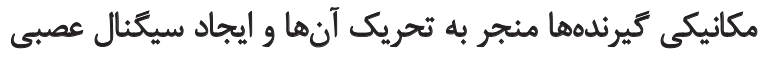

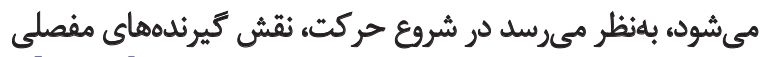

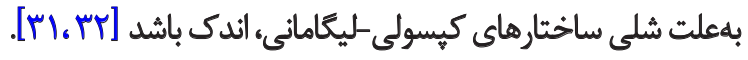

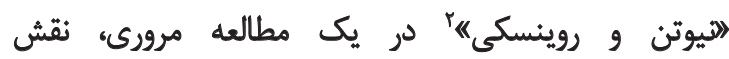

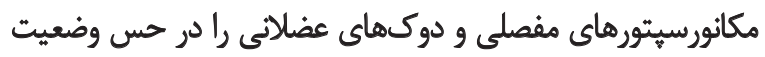

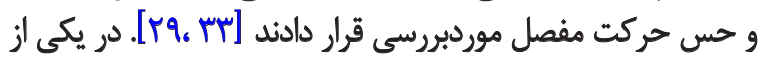

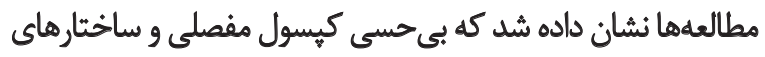

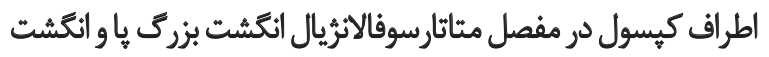

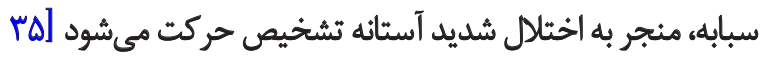

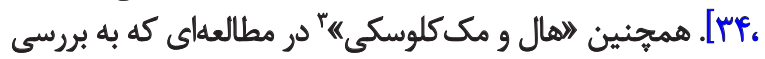

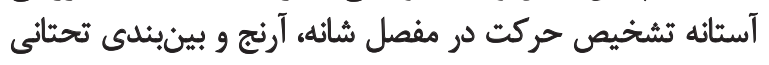

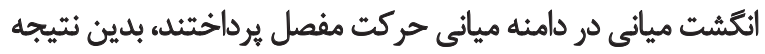

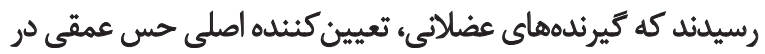

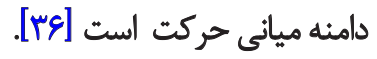

2. Newton and Rowinski

3. Hall and Mccloskey 


\section{References}

[1] Decoster LC, Vailas JC, Lindsay RH, Williams GR. Prevalence and features of joint hypermobility among adolescent athletes. Archives of Pediatrics \& Adolescent Medicine. 1997; 151(10):98992.

[2] Carter C, Wilkinson J. Persistent joint laxity and congenital dislocation of the hip. Journal of Bone \& Joint Surgery. 1964; 46(1):40-45.

[3] El-Garf A, Mahmoud G, Mahgoub E. Hypermobility among Egyptian children: prevalence and features. Journal of Rheumatology. 1998; 25(5):1003-005

[4] Beighton P, Solomon L, Soskolne C. Articular mobility in an African population. Annals of the Rheumatic Diseases. 1973; 32(5):413-18.

[5] Birrell F, Adebajo A, Hazleman B, Silman A. High prevalence of joint laxity in West Africans. Rheumatology. 1994; 33(1):56-59.

[6] Hakim A, Grahame R. Joint hypermobility. Best Practice \& Research Clinical Rheumatology. 2003; 17(6):989-004.

[7] Neumann DA. Kinesiology of the musculoskeletal system: foundations for rehabilitation. Philadelphia: Elsevier Health Sciences; 2013.

[8] Kirk J, Ansell B, Bywaters E. The hypermobility syndrome: Musculoskeletal complaints associated with generalized joint hypermobility. Annals of the Rheumatic Diseases. 1967; 26(5):419-25.

[9] Skoumal M, Haberhauer G, Mayr H. Concomitant diseases in primary joint hypermobility syndrome. Medizinische Klinik. 2004; 99(10):585-90

[10] Grahame R. The hypermobility syndrome. Annals of the Rheumatic Diseases. 1990; 49(3):199-200.

[11] Lippitt SB, Vanderhooft JE, Harris SL, Sidles JA, Harryman DT, Matsen FA. Glenohumeral stability from concavity-compression: a quantitative analysis. Journal of Shoulder and Elbow Surgery. 1993; 2(1):27-35.

[12] Nodehi Moghadam A, Kharazmi E. Comparision of shoulder proprioception between female volleyball players and non athlete femals. Journal of Rehabilitation. 2009; 10(2):45-49.

[13] Lephart SM, Pincivero DM, Giraido JL, Fu FH. The role of proprioception in the management and rehabilitation of athletic injuries. American Journal of Sports Medicine. 1997; 25(1):130-37.

[14] Hall JE. Guyton and Hall textbook of medical physiology. Philadelphia: Elsevier Health Sciences; 2010.

[15] Lohrer H, Alt W, Gollhofer A. Neuromuscular properties and functional aspects of taped ankles. American Journal of Sports Medicine. 1999; 27(1):69-75.

[16] Mallik A, Ferrell W, McDonald A, Sturrock R. Impaired proprioceptive acuity at the proximal interphalangeal joint in patients with the hypermobility syndrome. Rheumatology. 1994; 33(7):631-37.

[17] Yousefzadeh A, Khalkhali Zavieh M, Khademi K, Rahimi A. Studying the knee joint proprioception in generalized joint hypermobility as compared to healthy subjects. Journal of Research in Rehabilitation Sciences. 2012; 1(1):1-9.

$$
\begin{aligned}
& \text { حس عمقى مفصل ميشود كه خود ميتواند بهصورت يك } \\
& \text { جرخه ادامه يابد و منجر به شلى بيشتر شود [Tr] } \\
& \text { نتيجليَيرى }
\end{aligned}
$$

نتايج تحقيق نشان داد بين آستانه تشخيص حركت مفصل

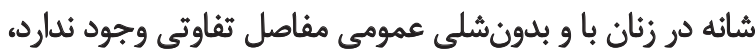

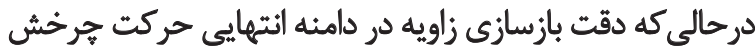

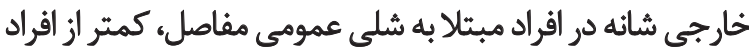

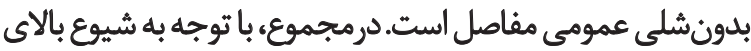

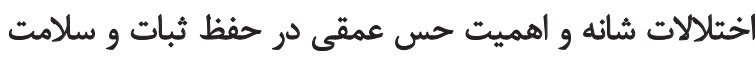

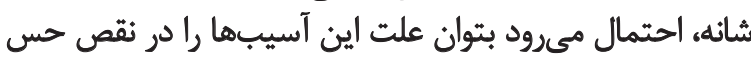

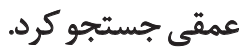

Lugsusen

دردسترسنبودن دستًاههايى با دقت بالاتر و مجرز به كيسه

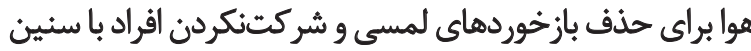

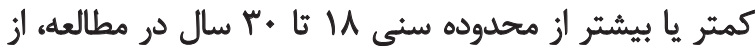
محدوديتهاي اين مطالعه محسوب مى مشود. |يشئهادها

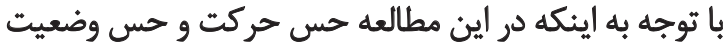

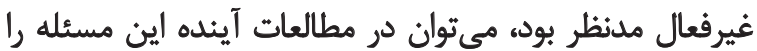

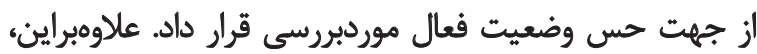

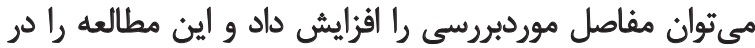

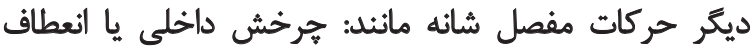

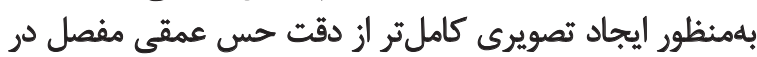
حركات عملكردى يا بعد از اجراى بروتكل خستخى انجى انجام داد.

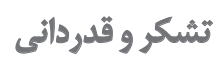

مقاله حاضر بركرفته از باياننامه كارشناسى ارشد خانم ماهنان

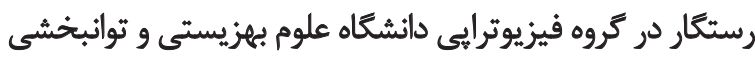

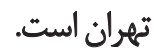


[18] Sahin N, Baskent A, Cakmak A, Salli A, Ugurlu H, Berker E. Evaluation of knee proprioception and effects of proprioception exercise in patients with benign joint hypermobility syndrome. Rheumatology International. 2008; 28(10):995-1000.

[19] Ferrell WR, Tennant N, Sturrock RD, Ashton L, Creed G, Brydson G, et al. Amelioration of symptoms by enhancement of proprioception in patients with joint hypermobility syndrome. Arthritis \& Rheumatism. 2004; 50(10):3323-328.

[20] Lephart SM, Warner JJ, Borsa PA, Fu FH. Proprioception of the shoulder joint in healthy, unstable, and surgically repaired shoulders. Journal of Shoulder and Elbow Surgery. 1994; 3(6):371-80.

[21] Allegrucci M, Whitney SL, Lephart SM, Irrgang JJ, Fu FH. Shoulder kinesthesia in healthy unilateral athletes participating in upper extremity sports. Journal of Orthopaedic \& Sports Physical Therapy. 1995; 21(4):220-26.

[22] Blasier R, Carpenter J, Huston L. Shoulder proprioception. Effect of joint laxity, joint position, and direction of motion. Orthopaedic Review. 1994; 23(1):45-50.

[23] Wilk KE, Andrews JR, Arrigo CA, Keirns MA, Erber DJ. The strength characteristics of internal and external rotator muscles in professional baseball pitchers. American Journal of Sports Medicine. 1993; 21(1):61-66.

[24] Lee HM, Liau JJ, Cheng CK, Tan CM, Shih JT. Evaluation of shoulder proprioception following muscle fatigue. Clinical Biomechanics. 2003; 18(9):843-47.

[25] Carpenter JE, Blasier RB, Pellizzon GG. The effects of muscle fatigue on shoulder joint position sense. American Journal of Sports Medicine. 1998; 26(2):262-65.

[26] Ellenbecker TS, Roetert EP. Testing isokinetic muscular fatigue of shoulder internal and external rotation in elite junior tennis players. Journal of Orthopaedic \& Sports Physical Therapy. 1999; 29(5):275-81.

[27] Joghatin Alibazi R, Bakhsi E, Nodehi Moghadam A, Zarabi V, Nakhaei N. [The effect of muscular fatigue on normal biomechanics of shoulder girdle: a systematic review of the literature (Persian)]. Journal of Rehabilitation. 2015; 16(3):242-51.

[28] Vangsness CT, Ennis M, Taylor JG, Atkinson R. Neural anatomy of the glenohumeral ligaments, labrum, and subacromial bursa. Arthroscopy: Journal of Arthroscopic \& Related Surgery. 1995; 11(2):180-84.

[29] Rowinski MJ. Afferent neurobiology of the joint. In: Gould JA, Davies GJ, editors. Orthopaedic and sports physical therapy. $2^{\text {nd }}$ ed. St. Louis: Mosby; 1985, p. 50-64.

[30] Clark FJ, Burgess P. Slowly adapting receptors in cat knee joint: can they signal joint angle? Journal of Neurophysiology. 1975; 38(6):1448-463.

[31] Rogol IM, Ernst G, Perrin DH. Open and closed kinetic chain exercises improve shoulder joint reposition sense equally in healthy subjects. Journal of Athletic Training. 1998; 33(4):315-18.

[32] Janwantanakul P, Magarey ME, Jones MA, Dansie BR. Variation in shoulder position sense at mid and extreme range of motion. Archives of Physical Medicine and Rehabilitation. 2001; 82(6):840-44.
[33] Newton RA. Joint receptor contributions to reflexive and kinesthetic responses. Physical Therapy. 1982; 62(1):22-29.

[34] Browne K, Lee J, Ring P. The sensation of passive movement at the metatarso-phalangeal joint of the great toe in man. Journal of Physiology. 1954; 126(3):448-58.

[35] Provins K. The effect of peripheral nerve block on the appreciation and execution of finger movements. Journal of Physiology. 1958; 143(1):55-67.

[36] Hall LA, McCloskey D. Detections of movements imposed on finger, elbow and shoulder joints. Journal of Physiology. 1983; 335(1):519-33.

[37] Iatridou K, Mandalidis D, Chronopoulos E, Vagenas G, Athanasopoulos S. Static and dynamic body balance following provocation of the visual and vestibular systems in females with and without joint hypermobility syndrome. Journal of Bodywork and Movement Therapies. 2014; 18(2):159-64. 